7th International Conference on Predictive Modelling of Food Quality and Safety

7ICPMF, September 12 - 15th, 2011, Dublin, Ireland Radisson Blu Royal Hotel, Golden Lane, Dublin 8, Ireland

Conference Proceedings

www.icpmf.org/2011

E. Cummins, J.M. Frías and V.P. Valdramidis (Eds.), Predictive Modelling of Food Quality and Safety - Conference Proceedings, UCD, DIT, Teagasc, Dublin, Ireland 


\title{
Meta-analysis for quantitative microbiological risk assessments and benchmarking data
}

\author{
H.M.W. den Besten, M.H. Zwietering \\ Wageningen University, Laboratory of Food Microbiology, Wageningen, The Netherlands \\ (heidy.denbesten@wur.nl,marcel.zwietering@wur.nl)
}

\begin{abstract}
Meta-analysis is an emerging approach in the food microbiology area to quantitatively integrate the findings of individual studies on kinetic parameters of interest. Meta-analyses provide global estimates of parameters with their variabilities, and give insight into the main influencing factors on microbiological kinetics. This paper discusses the opportunities of meta-analysis to generate sufficiently generic parameters - with their variability - for quantitative microbiological risk assessments, and demonstrates how the output of a metaanalysis can be used to benchmark future studies in order to position new data in perspective.
\end{abstract}

Keywords: QMRA, kinetics, global parameter estimates, variability, benchmarking

\section{Introduction}

Quantitative microbiological risk assessments (QMRAs) aim to quantify the risk related to the consumption of food products and include the assessment of the severity of a microbiological hazard (pathogens and/or toxins) and its likelihood of appearance (i.e. prevalence and concentration) (Lammerding, 1997). The four cornerstones of a QMRA are hazard identification, exposure assessment, hazard characterization, and risk characterization. During the exposure assessment a farm-to-fork approach can be applied, meaning that all steps of a food supply chain are quantitatively described in order to estimate the number of pathogens or the concentration of toxins at the moment of consumption. QMRAs are more and more used to set microbiological criteria and specifications in different steps of the food chain, to justify measures, to predict the effects of interventions, for food safety legislation, and to obtain insight into the most important phenomena responsible for the risks of foodborne diseases. One of the main practical difficulties of QMRAs is the need for an enormous amount of data for a wide range of kinetic parameters including their averages, variabilities and distributions. An emerging approach to gather global estimates of kinetic parameters with their associated variability is meta-analysis. A meta-analysis is a systematic analysis of a large collection of data from individual studies aiming to integrate the findings and to produce a global estimate of the effect of a particular intervention or treatment (Gonzales-Barron and Butler, 2011). Because a meta-analysis provides a quantitative summary of results over a broad range of individual studies, it allows not only to produce a global estimate of a parameter, but it also gives information about its variability and the sources of heterogeneity among the data of the individual studies. Moreover, the output of a meta-analysis can be used to benchmark future studies in order to position new data in perspective.

\section{Materials and Methods}

The meta-analysis approach can be used to address various questions where a reasonable amount of data exists. For several parameters needed to describe microbial behaviour, a literature search was performed and freely accessible data bases were searched. All quantitative data was structurally organized in databases on the question or parameter of interest to include quantitative and qualitative information of the individual studies. The data were then analysed for main explanatory factors and clustered to obtain global parameters (with their variability) and more specific parameters (with their variability). Furthermore the data bases were used to compare newly gathered data under specific conditions, to investigate the relevance of the effect of these conditions (benchmarking). 


\section{Results and Discussion}

\section{From a global to a product-specific parameter estimate}

Large data sets for irradiation parameter $D_{10}$ (Van Gerwen et al. 1999), concentration of contaminants in air in factory environments (Den Aantrekker et al. 2003), sedimentation velocities of micro-organisms (Den Aantrekker et al. 2003), heat inactivation parameters (Van Asselt and Zwietering, 2006), and high hydrostatic pressure inactivation parameters (Santillana-Farakos and Zwietering, 2011) were successfully gathered previously in our laboratory and evaluated. Main influencing factors could be identified and global and more specific estimations of the parameters with their attendant variabilities were estimated. The quantitative evaluation of the collated heat inactivation data resulted in global estimations of $D$-values and $z$-values for various foodborne pathogens. Many effects of factors reported to affect the $D$-value, such as $\mathrm{pH}$ and water activity $\left(\mathrm{a}_{\mathrm{w}}\right)$ of the food product, species and strain variability, were shown to be smaller in comparison with the variability of published $D$ values. Only a limited number of factors, including temperature, did have a significant effect on the $D$-values. For Listeria monocytogenes $967 D$-values were extracted from 14 individual studies and only the presence of salt $\left(10 \% \mathrm{w} / \mathrm{v}\right.$, or $\left.\mathrm{a}_{\mathrm{w}}<0.92\right)$ resulted in a significantly higher heat resistance. The upper limit of the $95 \%$ prediction interval (PI) of the $D$-values, estimated by integrating the data of all products excluding those with a low water activity (940 Dvalues), was used as a conservative estimation of the $D$-value (Table 1).

Table 1: $D$-values $\left(D_{\text {ref }}\right)$ at reference temperature $\left(T_{\text {ref }}\right)$ for Listeria monocytogenes in a wide variety of products (all products), and for a more specific product group (dairy) and product type (milk)

\begin{tabular}{llll}
\hline Product group & $D_{\text {ref }}(\min )^{\mathrm{a}}$ & $T_{\text {ref }}\left({ }^{\circ} \mathrm{C}\right)$ & $z\left({ }^{\circ} \mathrm{C}\right)$ \\
\hline All products & $0.273(0.045)$ & 72 & 7 \\
Dairy & $0.104(0.027)$ & 72 & 6.4 \\
Milk & $0.091(0.024)$ & 72 & 6.2 \\
\hline
\end{tabular}

${ }^{\bar{a}}$ Raw data were derived from the data set published by Van Asselt and Zwietering (2006) and represent the upper $95 \%$ prediction interval $D$-values, and the mean $D$-values between brackets.

When a large data base is available for a specific product or product group, then one can progress to a less conservative estimate which is based on a still sufficiently generic data set. The data set of Van Asselt and Zwietering (2006) included 280 D-values obtained in dairyrelated products including 226 from milk. These data were further analysed to derive global $D$-value estimates for dairy products and more specific also for milk (Table 1 ). The lower variability between individual studies resulted in smaller 95\% prediction intervals. Segregation by product type requires ample data sets to include variability between individual studies that can be attributed to factors such as experimental design, strain, laboratory, etc. When these data sets are available, then global estimates of parameters (with their variability) can be obtained for specific product groups.

\section{Benchmarking new data}

Both for heat inactivation and high hydrostatic pressure inactivation data, new literature information could be easily benchmarked using the data in the databases, showing the relevance of the studied effects in these studies. Van Asselt and Zwietering (2006) showed that the heat resistance of Salmonella species was neither significantly affected by product types or media nor by stain variability, $\mathrm{pH}$ and $\mathrm{a}_{\mathrm{w}}$ (1141 data extracted from literature). Only chocolate had a significant protective effect on Salmonella as inactivation in this product type resulted in remarkably high $D$-values and z-value (Figure 1). 


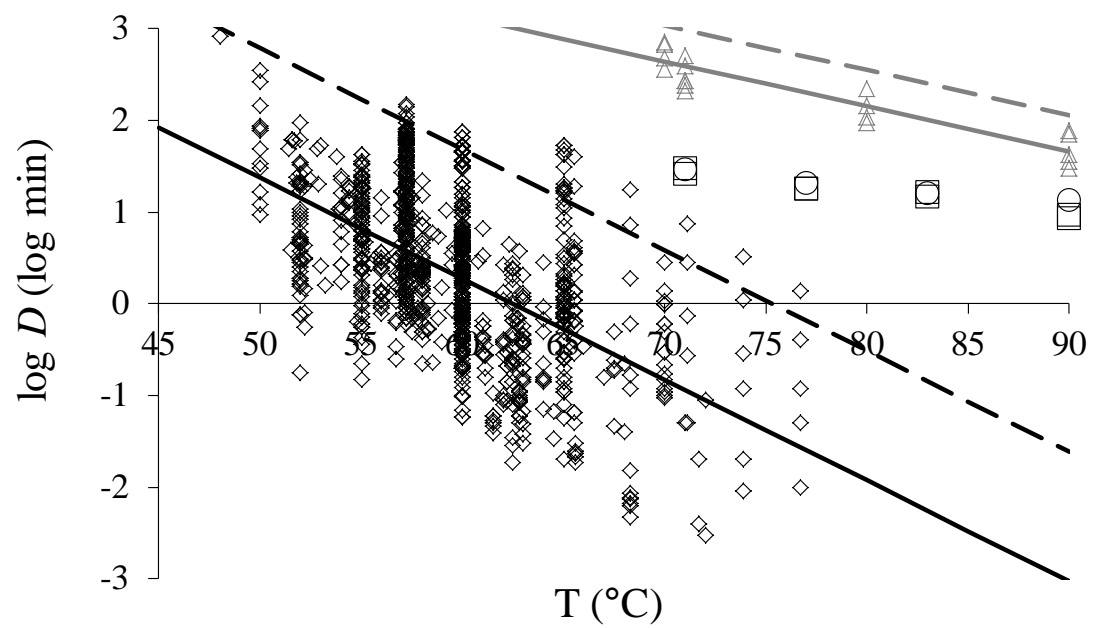

Figure 1: Heat resistance of Salmonella species in various food products and media $(\diamond)$, and in chocolate $(\triangle)$ as published by Van Asselt and Zwietering (2006). The solid line represents the linear regression of $\log D$ on the temperature and the dotted line represents the upper $95 \%$ prediction level. And the heat resistance of outbreak-associated Salmonella strains $(\bigcirc)$ and Salmonella strains obtained from other sources $(\square)$ in peanut butter as published by Ma et al. (2009).

This knowledge can be used to benchmark new published information. A recent publication reported the heat resistance of three sets of Salmonella strains in peanut butter, including strains isolated from patients associated with a peanut butter outbreak, culture collection strains and clinical isolates from sporadic cases (Ma et al. 2009). The heat resistance of the outbreak-associated strains were found to be more heat resistant at $90^{\circ} \mathrm{C}$ than the other Salmonella strains. However, when the $D$-values in peanut butter of the three different sets of strains were combined with the data set of Van Asselt and Zwietering (2006), then the heat resistance differences between the three sets of strains became - although significant - less obtrusive (Figure 1). The heat resistance of Salmonella in peanut butter was lower than those in chocolate as reported by Van Asselt and Zwietering (2006), but noticeably higher compared to inactivation in other product types. The product characteristics of peanut butter and chocolate - both high in fat content and low $\mathrm{a}_{\mathrm{w}}-$ might have contributed to the protective effect during heat inactivation in these product types. Because inactivation of Salmonella in both peanut butter and chocolate resulted in remarkably high $D$-values, it strengthened the separation of these product types from others, although these separative conclusions were based on a rather limited number of data collected for peanut butter (12 D-values) and for chocolate (20 D-values). The integration of new published data in an existing database can put new data in perspective, and highlights the prospects of meta-analysis to evaluate new data.

\section{Conclusions}

Meta-analysis is an emerging methodology in the area of food microbiology to systematically and critically collate a large number of individual studies and to quantitatively integrate their findings. When compiling comprehensive microbiological data from individual studies of different laboratories, several issues will be encountered. The overlap between individual studies can be rather limited as many differences can occur in the experimental set ups of individual studies, such as the strain(s) of choice and the intrinsic and extrinsic factors of the experiments. Therefore, often more than one variable has been changed between different studies, which make it unfeasible to quantify the effect of a single variable and urging to firstly focus on global estimation of parameters. Meta-analysis allows to quantitatively synthesize information and translate this into global parameter estimates and their 
corresponding variabilities. The differences between individual studies can contribute to rather high variabilities of parameter estimates, allowing to make conclusive separations of main effects. When ample data sets are available for a specific product types or effects, then progressing to a more detailed segregation level provides opportunities for less conservative estimations of parameters without forfeiting safety margins. The generation of sufficiently generic information, with its variability, is of importance to supply QMRAs with relevant data. Database building on the reviewed question or parameter reveals also a clear picture of the present knowledge, and can highlight areas where there is insufficient or an absence of information on factors that might affect the parameter of interest, and can therefore provide direction for future research. Moreover, systematically structured data bases on parameters give prospects to evaluate new published data, allowing to evaluate new findings and position them in perspective.

\section{References}

Den Aantrekker E.D., Beumer R.R., van Gerwen S.J.C., Zwietering M.H., van Schothorst M. and Boom R.M. (2003) Estimating the probability of recontamination via the air using Monte Carlo simulations. International Journal of Food Microbiology 87, 1-15.

Gonzales-Barron U. and Butler F. (2011) The use of meta-analytical tools in risk assessment for food safety. Food Microbiology 28, 823-827.

Lammerding A.M. (1997) An overview of microbial food safety risk assessment. Journal of Food Protection 60, 1420-1425.

Ma L., Zhang G., Gerner-Smidt P., Mantripragada V., Ezeoke I. and Doyle M.P. (2009) Thermal inactivation of Salmonella in peanut butter. Journal of Food Protection 72, 1596-1601.

Santillana-Farakos S.M. and Zwietering M.H. (2011) Data analysis of the inactivation of food-borne microorganisms under high hydrostatic pressure to establish global kinetic parameters and influencing factors. Submitted.

Van Asselt E.D. and Zwietering M.H. (2006) A systematic approach to determine global thermal inactivation parameters for various food pathogens. International Journal of Food Microbiology 107, 73-82.

Van Gerwen S.J.C., Rombouts F.M., van 't Riet K. and Zwietering M.H. (1999) A data analysis of the irradiation parameter D10 for bacteria and spores under various conditions. Journal of Food Protection 62, 1024-1032. 